\title{
EXPERIMENTAL EVALUATION OF THE SOLID PARTICLES BEHAVIOR IN A VERTICAL AIR FLOW BY USING IMAGING ANALYSIS
}

\author{
EMILIAN-FLORIN MOSNEGUTU ${ }^{1}$, NARCIS BARSAN ${ }^{1}$, ALEXANDRA-DANA \\ CHITIMUS $^{1}$, CLAUDIA TOMOZEI ${ }^{1 *}$, MIHAIL RISTEA ${ }^{2}$ \\ 1 "Vasile Alecsandri" University of Bacau, Department of Environmental and Mechanical \\ Engineering, Calea Marasesti 157, Bacau, 600115, Romania \\ ${ }^{2}$ UM 01961, Otopeni, 75100, Romania
}

\begin{abstract}
To analyse the behavior of a solid particle in a vertical ascending air flow a series of studies have been carried out, both theoretical and experimental. This article presents a new method of imaging analysis of the behavior of a solid particle, in order to extend this study through experimental applications. The working algorithm implies the analysis of images in different positions of the solid particle in the vertical ascending air flow, analysis in relation to a reference image. By using the mathematical apparatus, i.e. Mathcad software, the movement of the solid particle in the air flow has been emphasized.
\end{abstract}

Keywords: imaging analysis, solid particle, vertical ascending air flow

\section{INTRODUCTION}

To determine the behavior of a solid particle in different environments, different investigation method have been conceived. Recently, the practical study of the movement of a solid particle in an environment has been achieved due to technological and software development, such as the use of high-speed video cameras and special programs to process the footage $[1,2]$.

In some cases, the specialized programs, coming with high-speed cameras or conceived to analyse video files, are not adequate to analyse certain processes; this is why they different methods are conceived to process these files [3-5]. The specialized programs are generally to create an imaging analysis using certain bench-marks to be observed [1,2].

Experimental analysis uses two or more video cameras, or a single video camera and a laser system for highlighting the rotational motion of the solid particle or using radiolabeled particles. But there are situations in which the object pursued cannot be marked and that method of analyzing the movement film as such must be found. In this situation there are many industrial processes in which the movement of free bodies in different environments or on different work surfaces performs complex movements [1, 2, 6-10]. This article presents a new method for analyzing the behavior of a solid particle in an upward vertical air stream using different filtering and imaging methods existing in the Mathcad program.

\section{EXPERIMENTAL SETUP}

\footnotetext{
${ }^{*}$ Corresponding author, email: claudia.tomozei@ub.ro
}

(C) 2020 Alma Mater Publishing House 
To analyze the behavior of a solid particle in an ascending vertical air stream, a laboratory stand was used, the walls of which are made of transparent plexiglass, with a vertical air channel and three chambers of soothing. The stand works on the air discharge principle, the air feed being made by the lower side, through the feed hopper. The area used to analyze the behavior of the solid particle in the ascending vertical air channel is the cylindrical part of the laboratory stand (Figure 1).

In order to be able to analyze the behavior of a solid particle in the ascending vertical air channel, a HiSpec highspeed video camera was used, which was configured to make video recordings at 500 frames/second (Figure 1) [11].

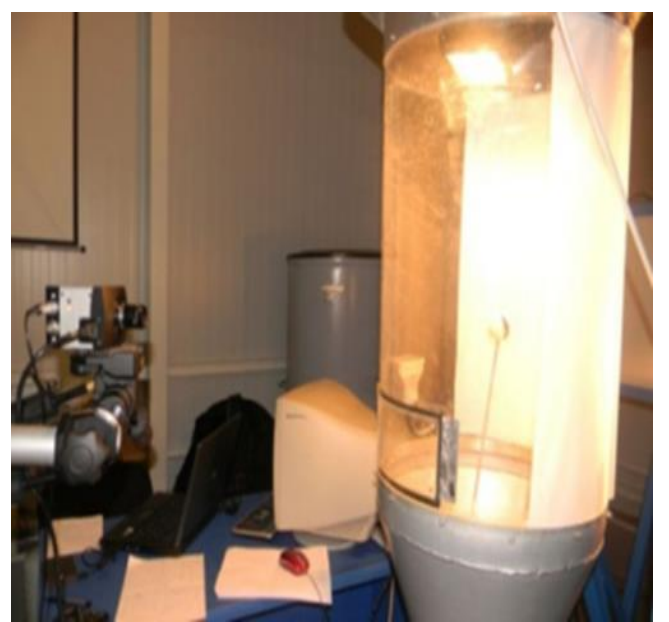

Fig. 1. The laboratory stand and position of the HiSpec high-speed camera [11].

Small particles could not be used as solid particles, as in the case of various experimental studies found in the literature, large polystyrene particles with dimensions of $\varnothing 27$ was use, due to the size of the air channel. Figure 2 presents a new imaging analysis methodology designed to highlight the free movement of the solid particle in the ascending vertical air channel (Figure 2) [11].

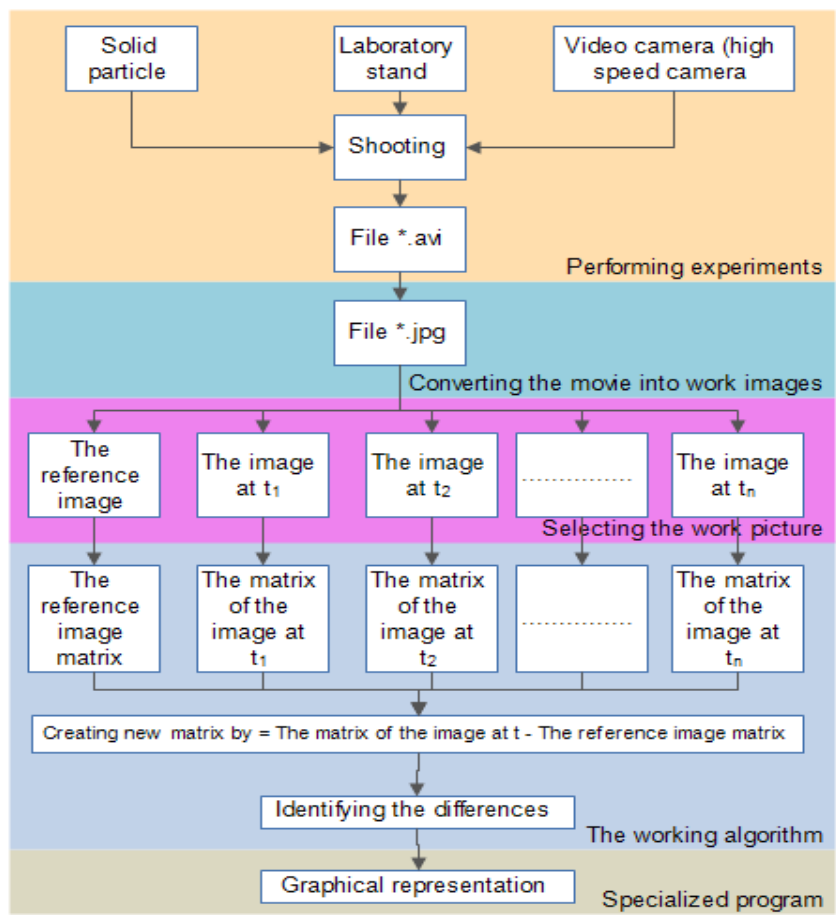

Fig. 2. Working method. 


\section{RESULTS AND DISCUSSION}

According to the working algorithm, a reference image with no solid particle has been chosen. This will be used to extract for the working images the background area of the concerned image, thus reducing the interferences that may appear due to color differences and reducing therefore the number of used colors (Figure 3).

To highlight the work stages, an image of time t4 (for example from a movie which contains 400 frames $t 4$ can corresponding to frame 169) was chosen (Figure 4).

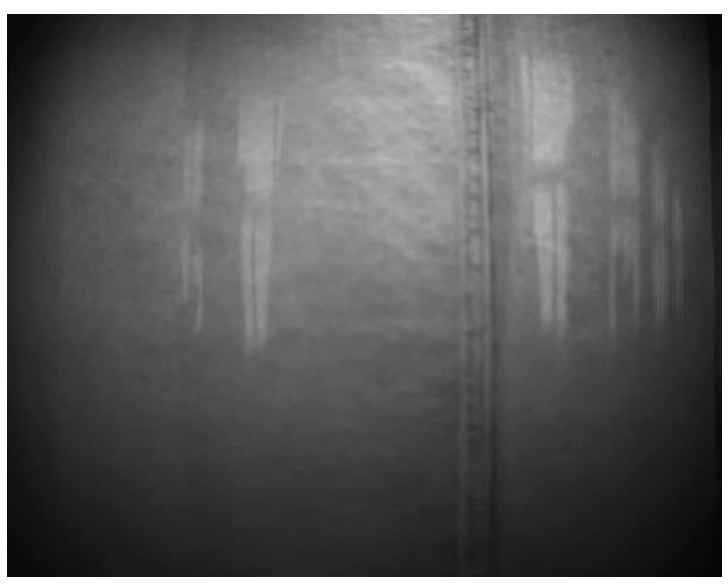

Fig. 3. Reference image.

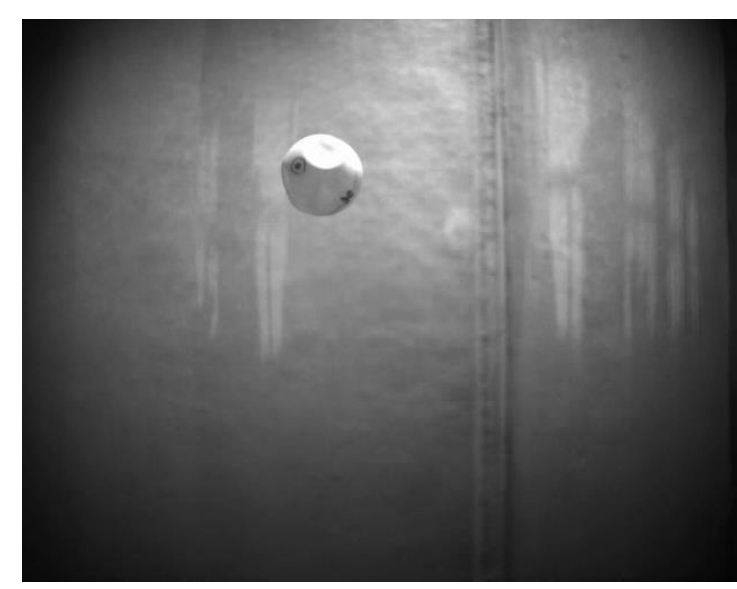

Fig. 4. Image subjected to analysis corresponding to time $\mathrm{t} 4$.

By making the difference between the image matrix corresponding to the time $t$ and the reference image, as has been stated above, the components from the studied image on the background are removed, thus remaining the different components from the picture, in this case the solid particle, $\mathrm{C} 5=\mathrm{a} 5 \mathrm{-a}$. For the realization of these work stages, as well as the future ones, the Mathcad program was used. Following the completion of the difference between the two arrays was obtained the following picture (Figure 5.)

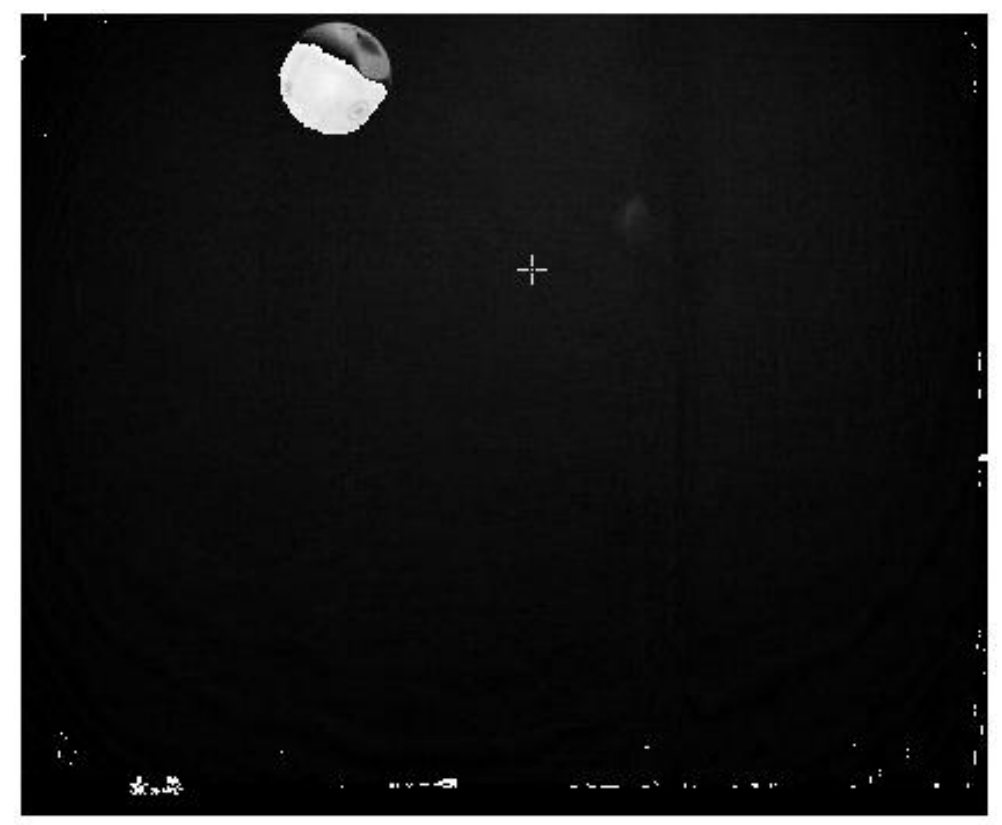

Fig. 5. Image obtained from preliminary processing.

To achieve greater image clarity, the following processing steps will be performed:

- The functions (1) and (2) are defined: 


$$
\begin{array}{r}
f 1(x)=i f(x<1,255,0) \\
f 2(x)=i f(x<100,0,255)
\end{array}
$$

where, $\mathrm{x}$ represents the working element, in this case is the matrix corresponding to the image; 255 and $0-$ represent the numerical data of the different colours, 255 for white and 0 for black; 1 and 100 - random values chosen to eliminate from the matrix the elements with the referred to values.

- $\quad$ In the functions defined as above are replaced the working matrices (equation (3) and Figure 6):

$$
d 1=\overrightarrow{f 1(a 1)} \text { and } d 5=\overrightarrow{f 1(a 5)}
$$

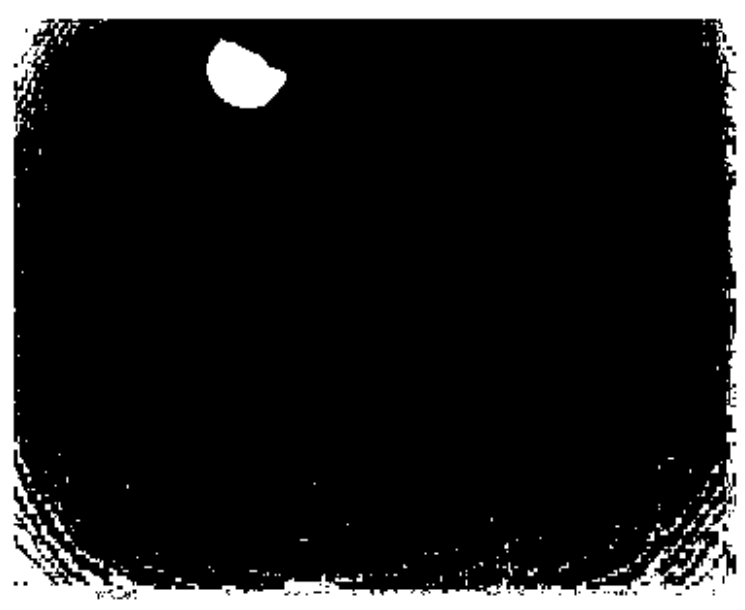

a)

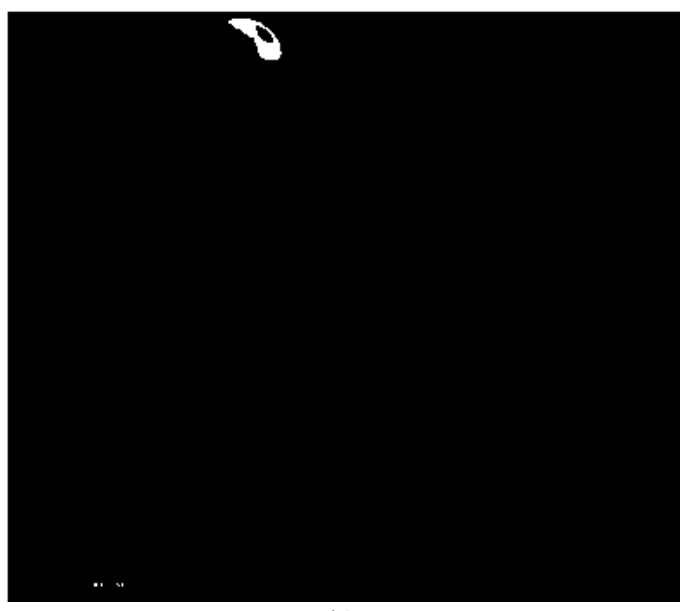

b)

Fig. 6. Images processed using the functions.

- $\quad$ By adding the two matrices thus obtained (Figure 7.a), the following image results (Figure 7.b);

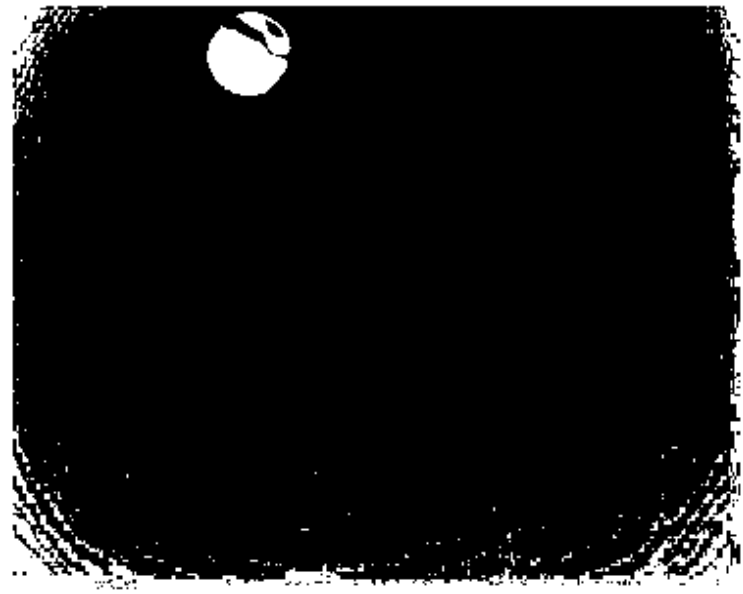

a)

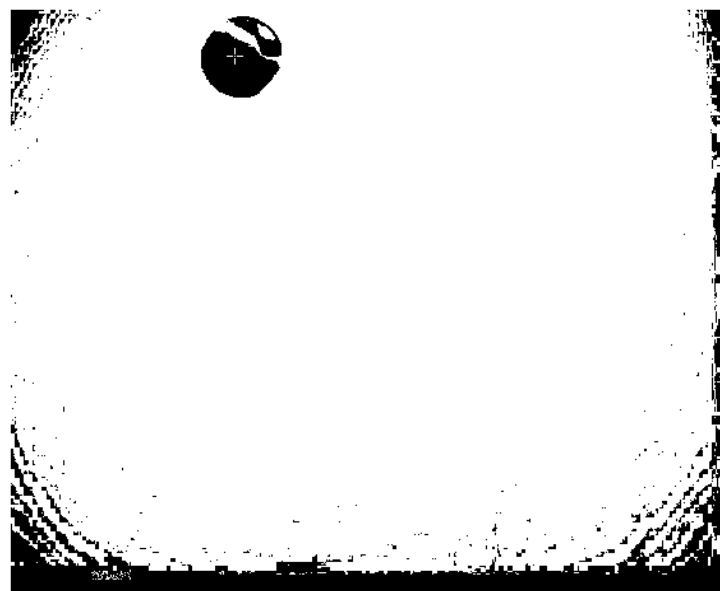

b)

Fig. 7. Images obtained by adding matrices.

By overlapping all the images, the trajectory that the solid particle has within the ascending vertical air channel can be obtained (Figure 8). 


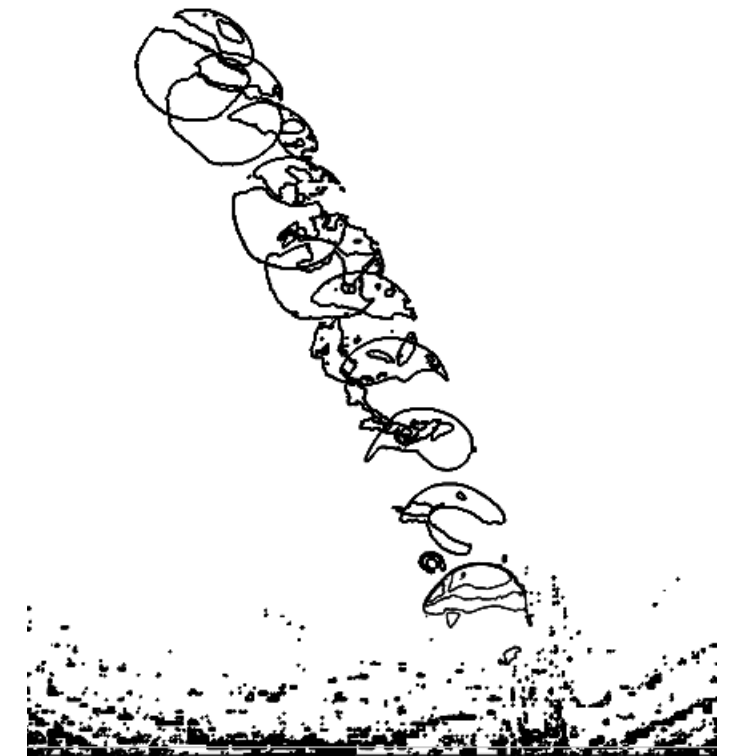

Fig. 8. Assembling the figures to identify the trajectory of the solid particle.

Because the particle under study has a spherical shape, it is desired to determine its size. To this end, the following work steps are performed:

1. Will be identified the number of columns and rows of the matrix under analysis (in this case the working matrix corresponding to the picture under analysis was denoted by de1). The cols and rows functions will be used for this (equations (4) and (5)):

$$
\begin{aligned}
& \operatorname{rows}(\operatorname{de} 1)=1001 \\
& \operatorname{cols}(\operatorname{de} 1)=880
\end{aligned}
$$

2. Two parameters will be defined, $i$ and $j$, which will vary in the range presented, the parameters that will be used to generate the circle used to determine the size of the solid particle (equations (6) and (7)):

$$
\begin{aligned}
& i=0 \ldots \operatorname{rows}(\operatorname{de} 1) \\
& j=0 \ldots \operatorname{cols}(\operatorname{de} 1)
\end{aligned}
$$

3. The circle diameter is defined, $r$. This will vary for each analyzed image, $r=6000$.

4. The coordinates of the center of the circle are defined, which must coincide with the middle of the studied body. These will have values that will damage for each analyzed image (equation (8)):

$$
\mathrm{cx}=100 \text { and } \mathrm{cy}=230
$$

5. The function to generate the circle is defined (equation 9).

$$
\mathrm{C} 1_{\mathrm{i}, \mathrm{j}}:=\mid \begin{array}{ll}
0 & \text { if }\left\lceil(\mathrm{i}-\mathrm{cx})^{2}+(\mathrm{j}-\mathrm{cy})^{2}\right\rceil \leq \mathrm{r} \\
1 & \text { otherwise }
\end{array}
$$

6. The two matrices overlap and adjustments of the above-mentioned parameter will be made so that the contour of the created circle overlaps the contour of the studied particle (Figure 9 and Figure 10). 


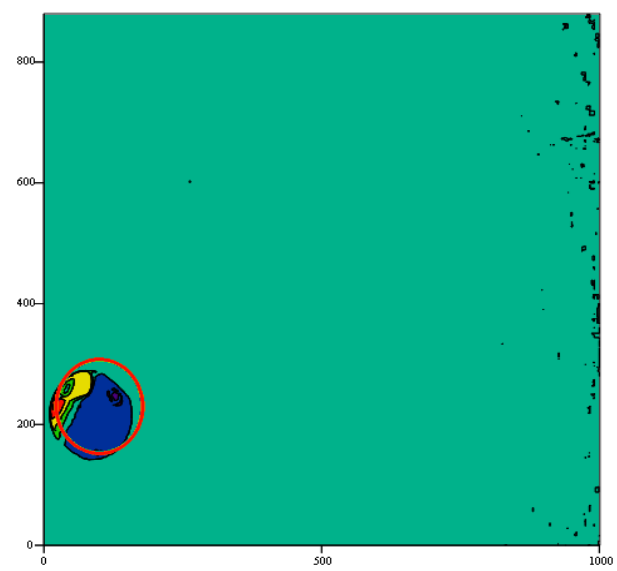

de1, C1

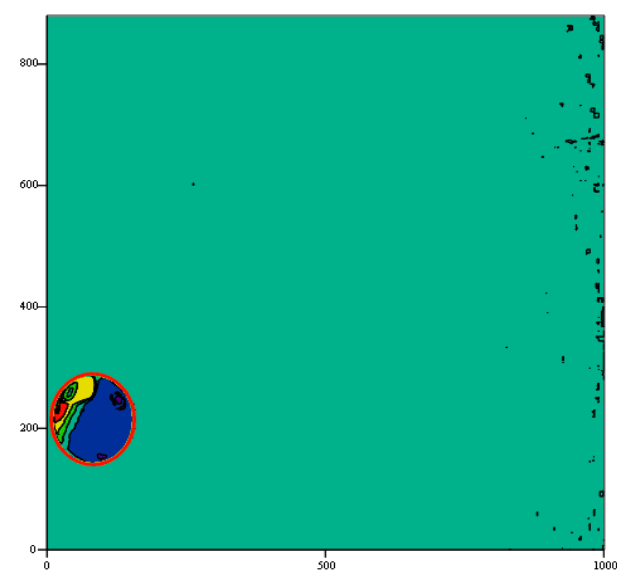

$\mathrm{de} 1, \mathrm{Cl}$

Fig. 9. Graphical representation of the overlap of the two matrices before adjustment.

Fig. 10. Graphical representation of the overlap of the two matrices after adjustment.

Table 1 shows the parameters for each picture analyzed.

Table 1. Variable values.

\begin{tabular}{|c|c|c|c|c|}
\hline No. & \multirow{2}{*}{ Picture } & Radium of the & \multicolumn{2}{|c|}{ the coordinates of the center of the circle } \\
\cline { 4 - 5 } & & circle, $\mathrm{r}$ & OX axes (cx) & OY axes (cy) \\
\hline 1 & de1 & 5500 & 82 & 215 \\
\hline 2 & de2 & 5400 & 143 & 257 \\
\hline 3 & de3 & 5300 & 209 & 296 \\
\hline 4 & de4 & 5200 & 283 & 336 \\
\hline 5 & de5 & 5100 & 353 & 382 \\
\hline 6 & de6 & 5000 & 435 & 422 \\
\hline 7 & de7 & 4900 & 525 & 462 \\
\hline 8 & de8 & 4800 & 605 & 502 \\
\hline 9 & de9 & 4700 & 714 & 534 \\
\hline 10 & de10 & 4600 & 820 & 565 \\
\hline 11 & de11 & 4500 & 920 & 602 \\
\hline
\end{tabular}

The new method for determining the behavior of a solid particle in an ascending vertical air channel involves the use of mathematical apparatus in order to highlight the motion of the studied particle. The newly created method can be used to analyze sound images in which different stages of the free movement of a solid particle in time are captured.

In general, the method can be applied to study the behavior of a single solid particle in different industrial processes in order to better understand these processes and to achieve their optimization. The presented method did not include the step of converting the sizes obtained from pixels into $\mathrm{mm}$.

From the image analysis carried out and as a result of measurements carried out it could plot graphically the path of the solid particles into the air channel (Figure 11).

Taking into account the fact that the particle analyzed in this working methodology moves from top to bottom, it is found that (from the analysis of the graphical representation, from Figure 11, and from the values described in Table 1) the solid particle moves away from the camcorder lens. 


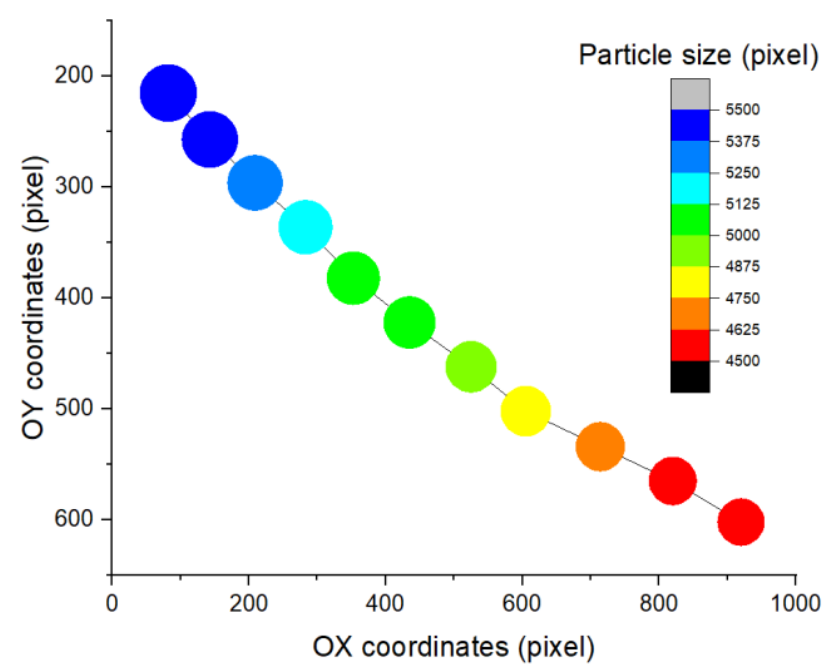

Fig. 11. Graphical representation of the path traveled by the solid particle in the air channel.

This methodology does not include scaling (conversion from pixel to $\mathrm{mm}$ ), a conversion that is specific to each type of particle used and the working environment (water or air). For this reason, it was not possible to identify the coordinate values for the $\mathrm{OZ}$ axis, respectively for the realization of a 3D representation.

\section{CONCLUSIONS}

In the literature are presented various studies that aim to track a solid particle or a mixture of solid particles in a fluid but very few of these studies present the methodology by which the images or films used for analysis are analyzed.

In order, to identify a method for tracking the behavior of solid particles in an environment, it was decided to create a methodology that is applied to the analysis of displacement for only one solid particle.

The methodology presented in this article involves the analysis of a sequence of pictures, a methodology that aims to highlight the behavior of a solid particle in an ascending vertical air stream.

Also, to obtain a clear trajectory of the solid particle the images used were processed as follows:

- An analysis of the image under study was made in relation to the basic image in order to eliminate the background elements.

- A series of filtering elements were made, from a mathematical point of view, which have the role of creating an image with two types of color, black and white.

- The images thus obtained were used to identify the location of the solid particle in the air duct and to determine the size of the solid particle.

The determination and identification of the variation of the solid particle size emphasizes that the identified method can be used to generate a 3D model.

The generated working methodology does not take into account the conversion of the sizes used, respectively pixels, into metric sizes, respectively $\mathrm{mm}$.

\section{REFERENCES}

[1] Bontas, O., Nedeff, V., Mosnegutu, E.F., Panainte, Study of factors influencing the solid particles on a flat inclined surface, Journal of Engineering Studies and Research, vol. 18, no. 1, 2012, p. 39.

[2] Bontas, O., Nedeff, V., Mosnegutu, E.F., Panainte, M., Irimia, O., Behaviour of solid particles on a flat oscillating surface, Environmental Engineering and Management Journal, vol. 12, no. 1, 2013, p. 17-22. 
[3] Do, Y.T., Tracking people in video camera images using neural networks, Advances in Intelligent Computing, Pt 1, Proceedings, vol. 3644, 2005, p. 301-309.

[4] Chavand, F., Colle, E., Chekhar, Y., N'zi, E.C., 3-D measurements using a video camera and a range finder, Ieee Transactions on Instrumentation and Measurement, vol. 46, no. 6, 1997, p. 1229-1235.

[5] Liu, W., Hu, C., He, Q., Wei, N., Meng, M.Q.H., 3D Optical Localization System Based on Four Video Cameras, Information-an International Interdisciplinary Journal, vol. 13, no. 6, 2010, p. 1909-1927.

[6] Souza, M.R., Pedrini, H., Digital video stabilization based on adaptive camera trajectory smoothing, Eurasip Journal on Image and Video Processing, vol. 37, 2018, p. 1-11.

[7] Gural, P.S., A new method of meteor trajectory determination applied to multiple unsynchronized video cameras, Meteoritics and Planetary Science, vol. 47, no. 9, 2012, p. 1405-1418.

[8] Prasertsakul, P., Kondo, T., Iida, H., Phatrapornnant, T., Camera operation estimation from video shot using 2D motion vector histogram, Multimedia Tools and Applications, 2020.

[9] Wu, Y.C., Wu, X.C., Yao, L.C., Grehan, G., Cen, K.F., Direct measurement of particle size and 3D velocity of a gas-solid pipe flow with digital holographic particle tracking velocimetry, Applied Optics, vol. 54, no. 9, 2015, p. 2514-2523.

[10] Theobalt, C., Albrecht, I., Haber, J., Magnor, M., Seidel, H.-P., Marks, J., Pitching a baseball - tracking highspeed motion with multi-exposure images, SIGGRAPH: International Conference on Computer Graphics and Interactive Techniques, 2004, p. 540-547.

[11] Ristea, M., Contributions to the study of aerodynamic separation process of solids particles mixture with application in food industry, PhD Thesis, (In Romanian), "Vasile Alecsandri” University of Bacau, 2014. 